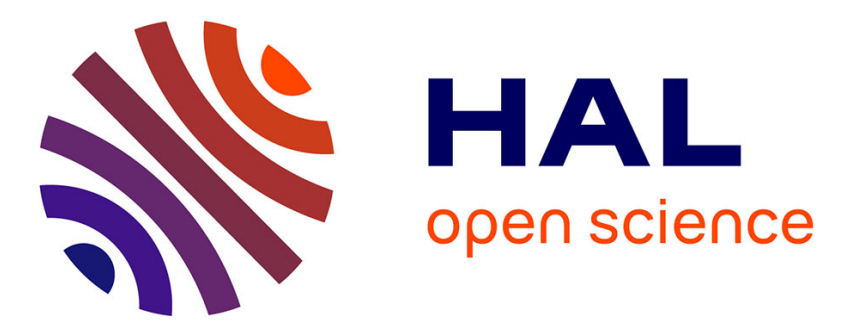

\title{
Identification de la réponse d'un capteur magnétique plat de proximité
}

\author{
P. Billaudel, J.C. Emond, V. Ménétrier, G. Villermain-Lecolier
}

\section{To cite this version:}

P. Billaudel, J.C. Emond, V. Ménétrier, G. Villermain-Lecolier. Identification de la réponse d'un capteur magnétique plat de proximité. Revue de Physique Appliquée, 1987, 22 (8), pp.779-783. 10.1051/rphysap:01987002208077900 . jpa-00245609

\section{HAL Id: jpa-00245609 https://hal.science/jpa-00245609}

Submitted on 1 Jan 1987

HAL is a multi-disciplinary open access archive for the deposit and dissemination of scientific research documents, whether they are published or not. The documents may come from teaching and research institutions in France or abroad, or from public or private research centers.
L'archive ouverte pluridisciplinaire HAL, est destinée au dépôt et à la diffusion de documents scientifiques de niveau recherche, publiés ou non, émanant des établissements d'enseignement et de recherche français ou étrangers, des laboratoires publics ou privés. 


\title{
Identification de la réponse d'un capteur magnétique plat de proximité
}

\author{
P. Billaudel, J. C. Emond, V. Ménétrier et G. Villermain-Lecolier \\ Laboratoire d'Application de la Microélectronique, Université de Reims, B.P. 347, 51062 Reims Cedex, \\ France
}

(Reçu le 26 février 1987, accepté le 30 avril 1987)

\begin{abstract}
Résumé. - Nous présentons un modèle électrique fournissant la réponse en fréquence de capteurs magnétiques plats constitués de deux spirales gravées sur les faces opposées d'un support diélectrique.
\end{abstract}

\begin{abstract}
We present an electrical model giving the frequency response for flat magnetic proximity sensors
\end{abstract} constituted of two spirals engraved on the opposite faces of a dielectric support.

\section{Introduction.}

De nombreux dispositifs de capteurs de proximité exploitant les courants de Foucault ont été décrits dans la littérature [1-4]. Dans des articles précédents [5] et [6] nous avons présenté les caractéristiques physiques d'un capteur magnétique de proximité constitué d'une spirale gravée sur un support époxy simple face. Nous avions alors montré que la réponse en fréquence d'un tel capteur était caractéristique d'un modèle inductance-résistance en série. Nous avions aussi montré, à travers deux montages expérimentaux, l'intérêt que présentent ces capteurs pour piloter le suivi d'un bord de tôle ou pour détecter la présence de trous dans une surface métallique. Ces deux exemples d'application exploitaient la modification d'impédance du capteur due à l'influence des courants de Foucault engendrés dans la cible par le champ du capteur.

De façon à améliorer les performances de ce type de capteurs, nous avons réalisé des échantillons gravés en double face, les deux spirales, de caractéristiques géométriques identiques, étant alors enroulées en sens contraire et reliées électriquement par leur centre; la technique de réalisation de ces capteurs procède des méthodes décrites dans [6].

Il est clair que, par rapport aux capteurs simple face de même géométrie, ces capteurs double face verront leur inductance quadruplée (en admettant un facteur de couplage égal à 1) et leur résistance doublée. Le présent article est consacré à l'identification de la réponse en fréquence de ces nouveaux capteurs.

\section{Caractérisation du capteur double face.}

2.1 MÉTHOdE UTILISÉE POUR L'IDENTIFICATION. - Le principe de l'identification du capteur consiste à calculer l'impédance $Z$ du modèle électrique proposé pour celui-ci, à simuler le diagramme de Bode théorique de ce modèle inséré dans un filtre passe-bas et à comparer le résultat obtenu au diagramme expérimental.

Le schéma du montage sera donc:

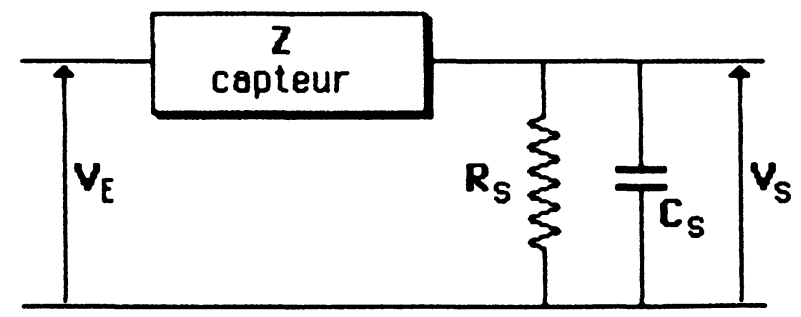

Fig. 1. - Montage pour l'identification.

[Identification apparatus.]

dans lequel $R_{\mathrm{S}}$ est la résistance de sortie et $C_{\mathrm{S}}$ représente les perturbations capacitives des appareils de mesure. La fonction de transfert de ce montage est donnée par:

$$
\frac{U_{\mathrm{S}}}{U_{\mathrm{E}}}=\frac{Z_{\mathrm{S}}}{Z_{\mathrm{S}}+Z} \quad \text { où } \quad Z_{\mathrm{S}}=\frac{R_{\mathrm{S}}}{p R_{\mathrm{S}} C_{\mathrm{S}}+1} .
$$

2.2 LE MODÈLE ÉLECTRIQUE DU CAPTEUR. - La présence de spires de part et d'autre de l'isolant 
induit inévitablement un effet capacitif, d'autant plus important que le capteur est de grandes dimensions, toutefois nous nous sommes limités à la seule contribution des spires en regard, une étude annexe ayant montré que la prise en compte des spires croisées n'améliorait que très légèrement les résultats tout en augmentant considérablement les temps de calcul.

Par contre, nous avons inclus la contribution des spires adjacentes sur une même face (leur influence est environ 10 fois plus faible que celle des spires en regard).

Compte tenu de ces remarques, le modèle électrique proposé pour le capteur sera :

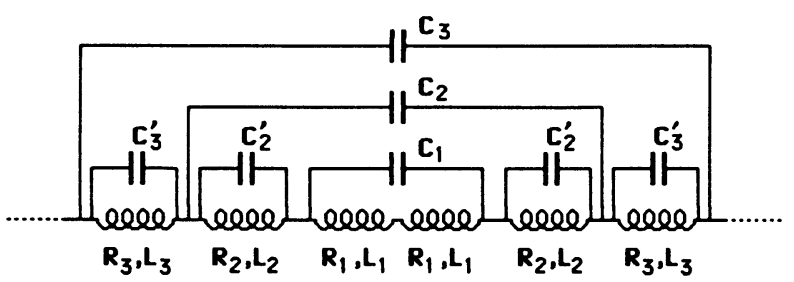

Fig. 2. - Modèle électrique du capteur.

[Electrical model for the sensor.]

dans lequel $L_{k}$ et $R_{k}$ représentent l'inductance et la résistance de la spire d'ordre $k$ (spire de rayon $\left.r_{k}\right), C_{k}^{\prime}$ (pour $k>1$ ) représente la capacité engendrée par les spires d'ordre $i$ et $i-1$ d'une même face et enfin $C_{k}$ représente la capacité engendrée par les deux spires d'ordre $k$ en regard.

Les calculs de ces éléments individuels sont explicités au paragraphe 3 .

Dans ce schéma, seule la cellule initiale $\left\{2 R_{1}\right.$; $\left.2 L_{1} ; C_{1}\right\}$ présente un cas particulier. Son impédance est :

$$
Z_{1}=\frac{2\left(p L_{1}+R_{1}\right)}{2 p C_{1}\left(p L_{1}+R_{1}\right)+1} .
$$

Ainsi, ayant calculé l'impédance $Z_{k}$ du sous-ensemble constitué des cellules jusqu'à l'ordre $k$, l'impédance $Z_{k+1}$ du sous-ensemble des cellules jusqu'à l'ordre $k+1$

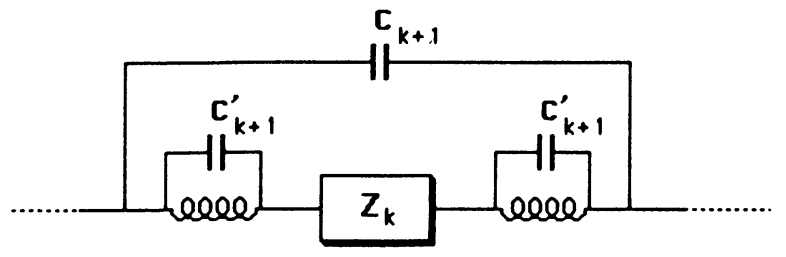

Fig. 3. - Sous-ensemble d'ordre $k+1$.

$[k+1$ order subset.]

sera donnée par :

$$
Z_{k+1}=\frac{Z_{k}+2 Z_{k+1}^{\prime}}{2 p Z_{k+1}^{\prime} C_{k+1}+p Z_{k} C_{k+1}+1}
$$

où $Z_{k+1}^{\prime}$ est l'impédance de la cellule élémentaire $\left\{R_{k+1} ; L_{k+1} ; C_{k+1}^{\prime}\right\}$, valant :

$$
Z_{k+1}^{\prime}=\frac{p L_{k+1}+R_{k+1}}{p^{2} L_{k+1} C_{k+1}^{\prime}+p R_{k+1} C_{k+1}^{\prime}+1} .
$$

\section{Calcul des éléments du modèle.}

La mise en œuvre des calculs numériques pour la simulation du modèle proposé nécessite la connaissance des capacités $C_{k}$ des spires en regard, des capacités $C_{k}^{\prime}$ des spires adjacentes et des inductances propres $L_{k}$ des spires considérées comme des bobines élémentaires isolées.

\subsection{CAlCul Des induCtanCES.}

3.1.1 Inductance propre des spires isolées. - Lors de notre étude sur les capteurs simple face nous avions mis au point une méthode de calcul des inductances dont nous avions pu vérifier la validité, aussi bien en confrontant les résultats théoriques avec les valeurs mesurées, qu'en les comparant avec ceux obtenus avec le logiciel FLUX-2D. Cette méthode est reprise dans les calculs relatifs aux capteurs double face.

Désignant par $r_{k}$ le rayon d'une spire, $e$ et $l$ l'épaisseur et la largeur du conducteur (rappelons que celui-ci est de section rectangulaire puisque les capteurs sont gravés sur epoxy ou sur kapton), si l'on pose :

$$
\rho=\sqrt{\frac{e l}{\pi}}
$$

l'inductance d'une telle spire est donnée par:

$$
\begin{aligned}
L_{k}=\mu_{0} & \left(\frac{r_{k}}{4}+\left(2 r_{k}-\rho\right) \times\right. \\
& \left.\times\left[\left(1-\frac{K_{k}^{2}}{2}\right) J_{1}\left[K_{k}\right]-J_{2}\left[K_{k}\right]\right]\right)
\end{aligned}
$$

où $J_{1}\left[K_{k}\right]$ et $J_{2}\left[K_{k}\right]$ sont les intégrales de Legendre de coefficient

$$
K_{k}=\frac{2 \sqrt{r_{k}\left(r_{k}-\rho\right)}}{2 r_{k}-\rho} .
$$

3.1.2 Inductance globale du capteur. - Bien que non nécessaire pour la simulation du modèle, nous avons établi l'inductance globale du capteur double face afin de pouvoir déterminer rapidement les géométries des échantillons présentant un rapport encombrement/performances optimal. Cette inductance $L$ est donnée par la relation classique (dans laquelle $N$ est le nombre de spires sur chacune des faces du capteur) :

$$
L=2\left(\sum_{k=1}^{N} L_{k}+\sum_{i=1}^{N} \sum_{\substack{j=1 \\ j \neq i}}^{N} L_{i j}+\sum_{i=1}^{N} \sum_{j=1}^{N} L_{i j}^{\prime}\right)
$$


où :

$L_{k}$ est l'inductance propre de la spire de rayon $r_{k}$, donnée par la formule (3.1) du paragraphe précédent.

$L_{i j}$ est le coefficient d'induction mutuelle entre les spires de rayons $r_{i}$ et $r_{j}$ situées sur une même face, donné par:

$L_{i j}=\mu_{0}\left(r_{i}+r_{j}\right)\left[\left(1-\frac{K_{i j}^{2}}{2}\right) J_{1}\left[K_{i j}\right]-J_{2}\left[K_{i j}\right]\right]$

$K_{i j}$ est le coefficient des intégrales de Legendre $J_{1}\left[K_{i j}\right]$ et $J_{2}\left[K_{i j}\right]$, valant ici :

$$
K_{i j}=\frac{2 \sqrt{r_{i} r_{j}}}{r_{i}+r_{j}}
$$

$L_{i j}^{\prime}$ est le coefficient d'induction mutuelle entre les spires de rayons $r_{i}$ et $r_{j}$ situées sur des faces différentes, donné par:

$$
\begin{aligned}
L_{i j}^{\prime}=\mu_{0} & \sqrt{\left(r_{i}^{2}+r_{j}^{2}\right)+E^{2}} \times \\
& \times\left[\left(1-\frac{K_{i j}^{\prime 2}}{2}\right) J_{1}\left[K_{i j}^{\prime}\right]-J_{2}\left[K_{i j}^{\prime}\right]\right]
\end{aligned}
$$

$K_{i j}^{\prime}$ est le coefficient des intégrales de Legendre $J_{1}\left[K_{i j}^{\prime}\right]$ et $J_{2}\left[K_{i j}^{\prime}\right]$, valant maintenant :

$$
K_{i j}^{\prime}=2 \sqrt{\frac{r_{i} r_{j}}{\left(r_{i}+r_{j}\right)^{2}+E^{2}}}
$$

et $E$ désigne l'épaisseur du diélectrique.

L'application de ces formules aux échantillons que nous avons testés donne des résultats tout à fait satisfaisants puisque l'erreur entre les valeurs calculées et les valeurs mesurées reste inférieure à $4 \%$, ainsi que le montre le tableau ci-dessous :

Tableau I. - Inductance calculée et mesurée de quelques échantillons.

[Calculated and measured impedance for some samples.]

\begin{tabular}{|l|c|c|l|}
\hline Diamètre intérieur $(\mathrm{mm})$ & 37,0 & 8,1 & 2,0 \\
Diamètre extérieur $(\mathrm{mm})$ & 50,0 & 17,0 & 10,0 \\
Nombre de spires & 19 & 19 & 40 \\
Epaisseur du diélectrique $(\mathrm{mm})$ & 1,6 & 1,6 & 0,125 \\
Largeur des spires $(\mu \mathrm{m})$ & 392 & 381 & 112 \\
Epaisseur des spires $(\mu \mathrm{m})$ & 35 & 35 & 35 \\
Inductance calculée $(\mu \mathrm{H})$ & 206,4 & 37,7 & 65,2 \\
Inductance mesurée $(\mu \mathrm{H})$ & 214,0 & 38,6 & 63,0 \\
\hline
\end{tabular}

L'obtention d'une largeur constante des spires au long d'une même bobine et la reproduction de bobines dont les spires ont une largeur rigoureusement identique posent des difficultés techniques de gravure, il était donc important de calculer l'incidence de la variation de la largeur du conducteur sur l'inductance de capteurs dont les autres caractéristiques géométriques seraient par ailleurs identiques.

Tableau II. - Influence de la largeur du conducteur sur l'impédance calculée.

[Influence of conductor width on the calculated impedance]

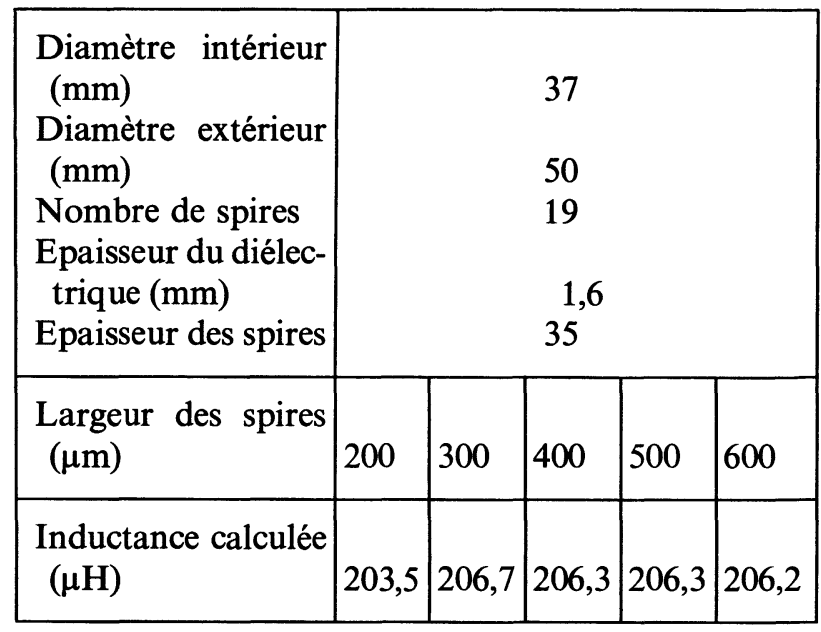

La variation maximale de l'inductance est de l'ordre de 1,5\%, donc négligeable, ainsi que l'on pouvait s'y attendre au vu de la faible importance du terme $\rho$ vis-à-vis du terme $2 r_{k}$ dans la relation (3.1a). Ce résultat nous permet donc de ne pas attacher une trop grande importance à la qualité de fabrication des capteurs.

3.2 CAlCul DES CAPACITÉs. - Désignant par $\Delta r$ le pas de la spirale d'Archimède que forment les spires du capteur et par $\varepsilon_{r}$ la conductivité relative du diélectrique $(5,22$ pour l'epoxy et 2,39 pour le kapton), les spires d'ordre $k$ en regard constituent un condensateur plan dont la capacité est :

$$
C_{k}=\frac{\varepsilon_{0} \varepsilon_{r} \pi l}{E}\left(2 r_{k}+\Delta r\right) \text {. }
$$

(Les paramètres $E$, $l$ et $r_{k}$ sont ceux introduits au paragraphe 3.1.)

Pour le calcul de la capacité $C_{k}^{\prime}$ formée par les spires adjacentes d'ordre $k-1$ et $k$ d'une même face, nous avons admis qu'elles constituaient un condensateur à armatures cylindriques, dont la valeur est :

$$
C_{k}^{\prime}=\frac{2 \pi \varepsilon_{0} e}{\ln \left(1+\frac{2 \Delta r}{2 r_{k}+l}\right)}
$$

Les valeurs numériques de ces capacités pour les géométries utilisées dans nos échantillons montrent que $C_{k}$ varie entre $1 \mathrm{pF}$ et $5 \mathrm{pF}$ alors que $C_{k}^{\prime}$ varie entre $0,1 \mathrm{pF}$ et $0,5 \mathrm{pF}$. 


\section{Résultats obtenus.}

Le modèle électrique proposé a été appliqué à de nombreux capteurs. Les résultats que nous présentons ici concernent l'échantillon double face de caractéristiques suivantes : diamètre extérieur $276 \mathrm{~mm}$, diamètre intérieur $104 \mathrm{~mm}$, 29 spires de largeur $2,5 \mathrm{~mm}$ et d'épaisseur $35 \mu \mathrm{m}$.

L'application des formules (2.1) pour une plage de fréquences comprise entre $0,1 \mathrm{MHz}$ et $10 \mathrm{MHz}$ donne le diagramme de Bode théorique ci-dessous :

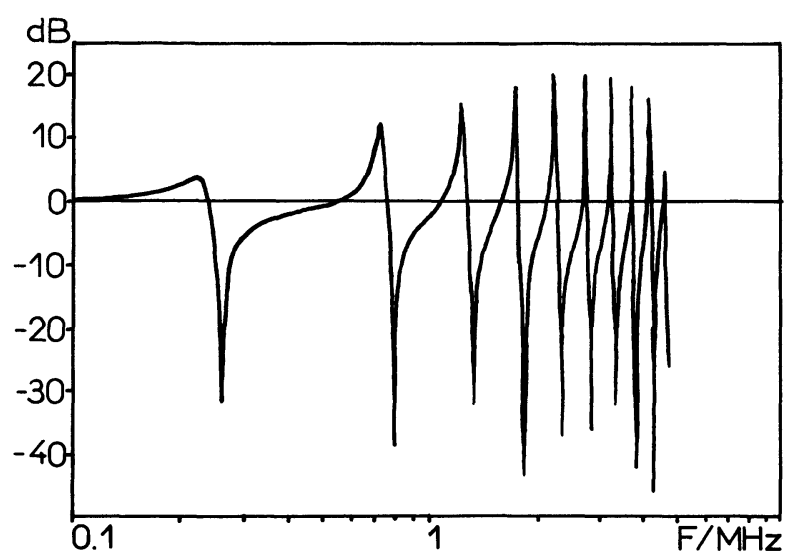

Fig. 4. - Diagramme de Bode théorique.

[Theoretical Bode diagram.]

Pour ce même échantillon, le diagramme de Bode relevé expérimentalement était :

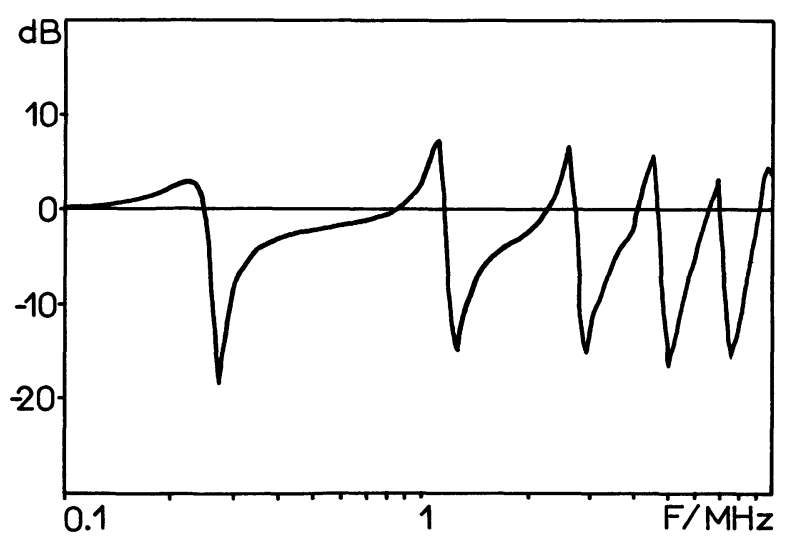

Fig. 5. - Diagramme de Bode expérimental.

[Expérimental Bode diagram.]

On remarque qu'au-delà du premier maximum, qui coïncide rigoureusement sur les deux tracés, la courbe théorique présente une compression en fréquence vis-à-vis de la courbe expérimentale.

L'analyse des valeurs numériques du rapport entre la fréquence expérimentale $F_{\mathrm{E}}$ et la fréquence théorique $F_{\mathrm{T}}$ aux points correspondant aux maxima du gain, met en évidence une progression arithméti- que de ce rapport dont la valeur initiale 1 correspond au premier maximum et dont la raison est 0,5 . Le tableau ci-dessous contient les rapports observés pour l'échantillon référencé ; la même constatation a pu être établie pour tous les échantillons que nous avons testés.

Tableau III. - Rapport entre la fréauence théorique et la fréauence expérimentale aux points maximaux du diagramme de Bode.

[Experimental and theoretical ratio at maximal points of the Bode diagram.]

\begin{tabular}{|l|l|l|l|l|l|l|}
\hline No du maximum & 1 & 2 & 3 & 4 & 5 & 6 \\
$F_{\mathrm{E}}(\mathrm{MHz})$ & 0,22 & 1,11 & 2,59 & 4,55 & 7,00 & 9,66 \\
$F_{\mathrm{T}}(\mathrm{MHz})$ & 0,22 & 0,74 & 1,25 & 1,78 & 2,25 & 2,76 \\
$F_{\mathrm{E}} / F_{\mathrm{T}}$ & 1,00 & 1,50 & 2,05 & 2,55 & 3,11 & 3,50 \\
\hline
\end{tabular}

La distorsion entre les valeurs théoriques et les valeurs expérimentales peut être attribuée à une non-linéarité qu'il faudrait introduire dans notre modèle. En tout état de cause et dans l'état actuel de nos investigations, la prise en compte du rapport $F_{\mathrm{E}} / F_{\mathrm{T}}$ nous permet d'exploiter un facteur de forme, valable quel que soit l'échantillon traité, fournissant la correction adéquate de la courbe théorique brute. La figure suivante est le diagramme de Bode théorique ainsi corrigé.

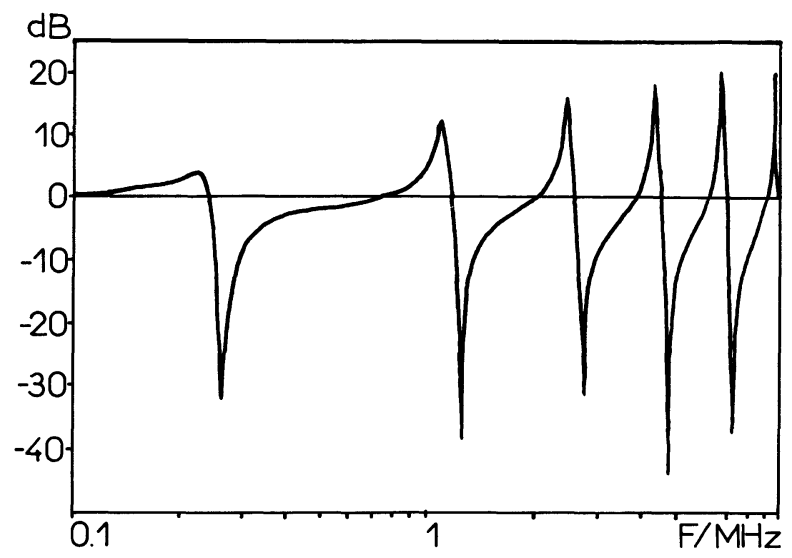

Fig. 6. - Diagramme de Bode théorique corrigé.

[Corrected theoretical Bode diagram.]

\section{Conclusion.}

Finalement, nous obtenons un modèle, corrigé en fréquence, donnant satisfaction. Evidemment, la correction sur le gain nécessiterait de connaître très précisément l'origine physique de la non-linéarité, afin de pouvoir l'introduire correctement dans le modèle.

Nous n'avons pas traité ce problème dans la mesure où seule la réponse en fréquence était un 
impératif dans la conception de nos capteurs. En effet, notre objectif était de déterminer rapidement les caractéristiques géométriques optimales de cap- teurs de positionnement; le modèle ainsi établi répond à cet objectif en nous permettant de prévoir les fréquences maximales de travail de ces capteurs.

\section{Bibliographie}

[1] AsCH, G., Les capteurs en instrumentation industrielle (Dunod, Paris) 1982.

[2] Garrat, J. D., J. Phys. E. 12 (1978) 563.

[3] PLACKO, D., Dispositif d'analyse de profil utilisant des capteurs à courant de Foucault. Thèse de troisième cycle. Orsay, 1984.

[4] Clergeot, H., Monteil, F., Placko, D., Detri' CHE, J. M., Dispositif d'analyse de profil utilisant des capteurs à courant de Foucault (AFCET Besançon) 1983.

[5] Villermain-Lecolier, G., Billat, A., SENSOR'85 - Transducer-Technik. Karlsruhe. Conference Proceedings (4) 1985, 621.

[6] Billat, A., Lecler, A., Emond, J. C., Villermain-Lecolier, G., Revue Phys. Appl. 21 (1986) 443-450. 